\title{
Head First
}

National Cancer Institute

\section{Source}

National Cancer Institute. Head First. NCI Thesaurus. Code C72676.

A direction of movement in which a person's head leads the way. 